\title{
Afferents from Vocal Motor and Respiratory Effectors Are Recruited during Vocal Production in Juvenile Songbirds
}

\author{
Sarah W. Bottjer and Michelle To \\ Section of Neurobiology, University of Southern California, Los Angeles, California 90089-2520
}

Learned behaviors require coordination of diverse sensory inputs with motivational and motor systems. Although mechanisms underlying vocal learning in songbirds have focused primarily on auditory inputs, it is likely that sensory inputs from vocal effectors also provide essential feedback. We investigated the role of somatosensory and respiratory inputs from vocal effectors of juvenile zebra finches (Taeniopygia guttata) during the stage of sensorimotor integration when they are learning to imitate a previously memorized tutor song. We report that song production induced expression of the immediate early gene product Fos in trigeminal regions that receive hypoglossal afferents from the tongue and syrinx (the main vocal organ). Furthermore, unilateral lesion of hypoglossal afferents greatly diminished singing-induced Fos expression on the side ipsilateral to the lesion, but not on the intact control side. In addition, unilateral lesion of the vagus reduced Fos expression in the ipsilateral nucleus of the solitary tract in singing birds. Lesion of the hypoglossal nerve to the syrinx greatly disrupted vocal behavior, whereas lesion of the hypoglossal nerve to the tongue exerted no obvious disruption and lesions of the vagus caused some alterations to song behavior. These results provide the first functional evidence that somatosensory and respiratory feedback from peripheral effectors is activated during vocal production and conveyed to brainstem regions. Such feedback is likely to play an important role in vocal learning during sensorimotor integration in juvenile birds and in maintaining stereotyped vocal behavior in adults.

\section{Introduction}

The production of learned vocal sounds requires precise coordination of vocal and respiratory effectors by a complex network of neural circuitry in both songbirds and humans (Goller and Cooper, 2004; Suthers and Zollinger, 2004; Wild, 2004). Multiple sensory inputs, including auditory, somatosensory, and visual, are necessary to learn and maintain production of stereotyped vocal utterances (Baptista and Morton, 1988; Morrison and Nottebohm, 1993; Suthers et al., 2002; Kuhl, 2004; Nasir and Ostry, 2009; Feng et al., 2011). In humans, somatosensory inputs from the vocal tract and facial skin are important for both perception and production of speech (Tremblay et al., 2003; Nasir and Ostry, 2006; Ito et al., 2009). For example, deaf subjects in whom somatosensory feedback is altered via small jaw displacements learn to correct their speech movements at the same rate as normal hearing subjects, suggesting that speakers use somatosensory as well as auditory representations of vocalizations for speech motor learning (Nasir and Ostry, 2008). Although sensory axons are known to innervate vocal effectors of songbirds, there is a dearth of evidence demonstrating a functional role for somatosensory

\footnotetext{
Received Feb. 29, 2012; revised May 29, 2012; accepted June 4, 2012.

Author contributions: S.W.B. designed research; M.T. performed research;S.W.B. and M.T. analyzed data; S.W.B. wrote the paper.

This work was supported by NIH Grant NS037547. We thank Martin Wild and Jenny Achiro for comments on this manuscript.

The authors declare no competing financial interests.

Correspondence should be addressed to Sarah Bottjer, 3641 Watt Way, Neurobiology, HNB 218, University of Southern California, Los Angeles, CA 90089-2520. E-mail: bottjer@usc.edu.

DOI:10.1523/JNEUROSCI.0990-12.2012

Copyright $\odot 2012$ the authors $\quad 0270-6474 / 12 / 3210895-12 \$ 15.00 / 0$
}

inputs in regulating song behavior (Bottjer and Arnold, 1984; but see Suthers et al., 2002).

Previous studies have shown that the act of singing in adult songbirds induces high levels of immediate early gene (IEG) expression in telencephalic vocal-control brain nuclei, even in the absence of auditory feedback (Jarvis and Nottebohm, 1997; Jin and Clayton, 1997; Kimpo and Doupe, 1997; Jarvis et al., 1998). Here we used the functional expression of an IEG to test whether sensory axons from vocal effectors of juvenile songbirds are activated as they are engaged in vocal learning (cf. Mongeau et al., 2003). The syrinx is the main vocal organ in songbirds; it comprises a group of muscles surrounding the bifurcation of the trachea into the two bronchi as well as extrinsic muscles attaching to the trachea and sternum. The role of the tongue in song production is uncertain, although tongue movements cause changes in both frequency and amplitude of emitted sounds in parrots (Beckers et al., 2004). In songbirds, lingual afferents have been thought to be more involved with feeding rather than vocalizing, whereas inputs from the syrinx have been shown to contribute strongly to song behavior (Nottebohm, 1971; Nottebohm and Nottebohm, 1976; Nottebohm et al., 1979; Wild, 1990). The role of other vocal effectors has been less well studied (but see Fletcher et al., 2006; Riede et al., 2006).

We investigated whether sensory feedback from vocal-respiratory effectors contributes to vocal production in juvenile birds engaged in sensorimotor integration by examining protein expression of the IEG Fos within brainstem regions that receive afferent inputs from the tongue, syrinx, and respiratory system via the hypoglossal and vagal nerves (Fig. 1). Elimination of hypoglossal afferents on one side diminished singing-induced Fos expression in ipsilateral tri- 
geminal regions, whereas unilateral lesion of the vagus decreased Fos expression in the ipsilateral solitary complex. The results argue that feedback from vocal and respiratory effectors plays a functional role during song learning, suggesting that sensorimotor integration requires nonauditory as well as auditory feedback.

\section{Materials and Methods}

Subjects and vocal recording. Zebra finches were bred and raised by their parents in our group breeding aviaries. All procedures were performed in accordance with protocols approved by the University of Southern California Institutional Animal Care and Use Committee and conformed to national regulatory policies. Juvenile male zebra finches were removed from the group breeding aviaries at $35 \mathrm{~d}$ of age and placed in individual cages in sound-isolated recording chambers. Song behavior was recorded digitally every few days until $\sim 55 \mathrm{~d}$ of age using AudioTechnica microphones (model P51680) and automated recording software (Sound Analysis Pro with a Firepod PreSonus sound card, 44 $\mathrm{kHz}$ ) (http://ofer.sci.ccny.cuny.edu/sound_ analysis_pro) (Tchernichovski et al., 2000).

Surgical procedures: nerve sections. Figure 1 shows schematically the arrangement of relevant nerves and their central terminations. The hypoglossal nerve of both birds and mammals contains sensory afferents (red) which diverge from motor axons (gray) at the X-XII (vagalhypoglossal) anastomosis and enter the brainstem along with the vagus. Hypoglossal sensory axons from both the tongue and syrinx of songbirds have cell bodies located in the jugular ganglion and terminate centrally in parts of the trigeminal complex including the principal sensory nucleus of $\mathrm{V}(\mathrm{PrV})$ and the nucleus of the descending trigeminal tract (nTTD) (Wild, 1981, 1990; Bottjer and Arnold, 1982). Unpublished work by J. M. Wild indicates that syringeal hypoglossal afferents also terminate in the nucleus of the solitary tract (Wild, 2004). The sensory terminal fields of the tongue and syrinx within the trigeminal complex have not been separately mapped. Traditionally, the tracheosyringeal branch of the hypoglossal nerve has been thought to contribute to song behavior, whereas the lingual branch has been thought to be more involved with feeding (although no direct tests of lingual nerve function in vocal behavior of songbirds have been reported; Nottebohm, 1971; Nottebohm and Nottebohm, 1976; Nottebohm et al., 1979; Wild, 1990).

Surgery was performed under a dissecting microscope on birds anesthetized via intramuscular injection of $\sim 0.05 \mathrm{ml}$ of Equithesin or with isoflurane gas ( $\sim 1.5 \%$, inhalation). The mean age of birds at the time of surgery was $55.6 \mathrm{~d}$ (range $=49-62 \mathrm{~d}$ ), which corresponds to a time of active sensorimotor integration when birds are singing at a high rate (Johnson et al., 2002). A lateral incision was made in the skin covering the ventral neck area overlying the hyoid muscle, and the hyoid and adjacent neck muscles were retracted to reveal the anastomosis of the main hypoglossal nerve trunk (XII) with the vagus (X). Both the tracheosyringeal and lingual branches of the hypoglossal nerve $\left(\mathrm{XII}_{\mathrm{ts}}\right.$ and $\mathrm{XII}_{\mathrm{l}}$, respectively; Fig. 1) include motor (gray) and sensory (red) axons. These two branches join to form the main XII trunk, which forms an anastomosis with the vagus at the point where the hypoglossal motor roots enter the main XII trunk. These motor roots emanate from hypoglossal motor neurons $\left(\mathrm{nXII}_{\mathrm{ts}}\right.$ and $\mathrm{nXII}_{1}$, for tracheosyringeal and lingual motor nuclei, respectively) and exit the brain ventrally. The vagus also contains both sensory (green) and motor (brown) axons, which join the hypoglossal afferent axons to form the X-XII trunk proximal to the anastomosis. Thus, the X-XII trunk contains sensory axons of the hypoglossal nerve (both lingual and tracheosyringeal) as well as sensory and motor axons of the vagal nerve, but does not include motor XII axons. We refer to this part of the nerve as sXII + X throughout the paper.

Six experimental birds underwent section of the sXII + X nerve just proximal to the anastomosis (Fig. 1c); this manipulation leaves the motor XII roots intact, but lesions sensory afferents from the tongue and syrinx, as well as vagal sensory and motor axons. Nerve sections were made by cutting the nerve with a pair of microscissors just proximal to the anastomosis on the left $(n=5)$ or right $(n=1)$ side. The majority of sections were made on the left side because close proximity of blood vessels prohibited access to the anastomosis on the right side in most cases. In two additional birds, the vagus joined the main trunk of XII proximal to the anastomosis such that it was possible to section the XII afferent fibers only, leaving the X axons intact (Bottjer and Arnold, 1984). Therefore, in these two birds, only the hypoglossal afferent axons were cut (sXII). The skin incision was closed with Collodion (Sigma) following surgery, and birds were returned to their recording box. Song behavior was recorded for 2-4 d postsurgery, up until the time birds were processed for Fos immunohistochemistry.

Various other nerves were sectioned in different groups. The lingual $\left(\mathrm{XII}_{1}\right)$ nerve was sectioned in four birds and the $\mathrm{XII}_{\mathrm{ts}}$ nerve was sectioned in four birds (Fig. 1 $a, b$ ). In both of these groups unilateral nerve sections were made slightly distal to the bifurcation of the main XII trunk into lingual and tracheosyringeal branches, such that both motor and sensory axons were cut in each branch. The vagus (X) was cut unilaterally in an additional four birds (Fig. 1d), including both sensory and motor axons. Sensory vagal axons terminate primarily in the nucleus of the solitary tract (nTS), and vagal efferents emanate from the motor nucleus of the vagus (nX; Fig. 1) No nerve section was performed on five singing birds (No Sxn/Singing); these birds did not receive any surgical procedures. We also included two non-singing control conditions. In the first group, four birds underwent unilateral section of the sensory XII axons plus the vagus (sXII + X/Non-singing), as described above for experimental birds. In the second group, four non-singing birds received no section 
(and no surgical procedures; No Sxn/Non-singing). Birds in both of these non-singing groups were left in their home cage but removed from their recording chambers immediately after lights on and transferred to the main laboratory to prevent them from singing before perfusion (see below).

The final $n$ values for each group varied by brain region due to tissue damage in some areas during processing; all nerve sections were made on the left side unless specified otherwise. Values for one bird in the sXII + $\mathrm{X}$-Singing group were 2 SDs above the mean, and this bird was eliminated as an outlier. For nTTD in Singing birds: $\mathrm{XXII}+\mathrm{X}(n=5 ; 1$ right $)$, sXII only $(n=2), \mathrm{X}(n=4), \mathrm{XII}_{\mathrm{ts}}(n=3 ; 2$ right $), \mathrm{XII}_{1}(n=4)$, No Sxn $(n=5)$. For PrV in Singing birds: sXII $+\mathrm{X}(n=4$; 1 right $)$, sXII only $(n=$ $1), \mathrm{X}(n=3), \mathrm{XII}_{\mathrm{ts}}(n=3 ; 2$ right $), \mathrm{XII}_{1}(n=3)$, No Sxn $(n=4)$. For $\mathrm{nTS}$ in Singing birds: $\mathrm{sXII}+\mathrm{X}(n=5 ; 1$ right $), \mathrm{X}(n=4), \mathrm{XII}_{\mathrm{ts}}(n=4 ; 2$ right $)$, $\mathrm{XII}_{1}(n=4)$, No Sxn $(n=5)$. For Non-singing birds: sXII $+\mathrm{X}(n=4$ for all three nuclei), No Sxn ( $n=4$ in both nTTD and PrV and $n=5$ in nTS).

Singing, perfusion, and immunohistochemistry. Two to $4 \mathrm{~d}$ postsurgery, birds were allowed to sing in the morning at lights on until at least $10 \mathrm{~min}$ of song behavior was recorded. Singing birds were anesthetized and perfused $\sim 60 \mathrm{~min}$ (range $=30-120 \mathrm{~min}$ ) after the onset of singing. Nonsinging control birds were removed from their recording chamber immediately after lights on and transferred to the main laboratory, where they were observed before being anesthetized for 15-20 min (one bird was observed for a period of $1 \mathrm{~h}$ ). The experimenter tapped the cage or waved at birds to prevent them from singing if necessary. All birds were deeply anesthetized with $0.08-0.10 \mathrm{ml}$ of Equithesin and perfused through the heart with $9 \mathrm{ml}$ of $0.02 \mathrm{M}$ PBS followed by $35 \mathrm{ml}$ of a $4 \%$ paraformaldehyde plus $0.1 \%$ glutaraldehyde. Brains were postfixed for $12-18 \mathrm{~h}$ in $4 \%$ paraformaldehyde, cryoprotected in $25 \%$ sucrose, and frozen-sectioned in the coronal plane at a thickness of $50 \mu \mathrm{m}$ into two or three series: two free-floating series were used for ZENK and Fos immunohistochemical staining, and a third was Nissl-stained. Because staining for ZENK was inconsistent in the hindbrain, we have omitted a discussion of those results in the present study.

To detect Fos protein, free-floating tissue sections were incubated in $1 \% \mathrm{H}_{2} \mathrm{O}_{2}$ to block endogenous peroxidase before being incubated in blocking serum ( $5 \%$ normal goat serum). Sections were then incubated overnight in primary antibody to Fos (sc-253 rabbit IgG; Santa Cruz Biotechnology). Approximately 4-6 control sections (distributed along the rostral-caudal axis) were removed and set aside in PBS during the incubation period. The next morning, the tissue was incubated in biotinylated goat anti-rabbit IgG and avidin-biotin peroxidase reagent (ABC Elite Kit, Vector Laboratories). Antibody labeling was visualized with $0.05 \% 3,3$ '-diaminobenzidine solution; the concentration of $\mathrm{H}_{2} \mathrm{O}_{2}$ was increased incrementally from $0.003 \%$ to $0.015 \%$ to enhance the sensitivity of labeling. Sections were washed with $0.02 \mathrm{M}$ PBS solution between incubations at room temperature. Sections were then mounted on glass slides coated with gelatin and coverslipped with Permount. Control sections that were not exposed to the primary antibody never showed positive staining.

Quantification. We quantified Fos expression without knowledge of the experimental treatment received by any bird using the Virtual Slice module of Neurolucida (MicroBrightField). Briefly, image montages of the entire medulla at levels containing PrV, nTTD, and the nTS were captured in Neurolucida using a digital camera (MicroFire CX9000, Optronics) interfaced with a Leica DMRE microscope with a motorized stage (10× objective) and saved as an 8-bit image. Contours were drawn around each of these nuclei in each section in which they appeared, and the number of Fos + neurons within each contour on the left and right sides was quantified separately. The only parameter that varied between animals and sections in the Neurolucida software was the threshold used to highlight Fos + cells; the grayscale value above which a given cluster of pixels was considered to be signal was set for each section by matching the highlighted pixels to correspond as closely as possible to labeled cells. In some cases a higher background or lower signal yielded inaccurate counts using this strategy. In such cases where the signal-to-noise ratio was lower, we manually added markers over labeled cells that were not detected by thresholding to include them with the thresholded number of cells. The accuracy of cell counts obtained using this automated proce- dure was thus verified by manual inspection of labeled cells on every section that was counted. Immunoreactive cells varied somewhat in their staining intensity, and we followed the procedure of previous papers in counting all clearly labeled cells (Mello and Ribeiro, 1998; Whitney et al., 2000). The absolute number of Fos + neurons counted for each animal was divided by the volume of tissue measured to yield a density measure (number $/ \mathrm{mm}^{3}$ ) for left versus right sides of the brain. A Fos density ratio was computed by dividing the density of Fos + cells on the side with a nerve section by the density of Fos + cells on the intact side (in control birds with no nerve section this ratio was computed by dividing the density of Fos + cells on the left side by that on the right side). This ratio yields a value of 1.0 if the nerve section has no effect on singing-induced Fos expression, and a lower number if the nerve section diminishes Fos expression on the side with a nerve section. The density of Fos + neurons on the intact side in Singing versus Non-singing birds was used to provide an estimate of the level of singing-induced Fos induction.

We examined Fos expression in the song motor-control nuclei RA (robust nucleus of the arcopallium) and HVC (High Vocal Center) qualitatively and assigned a number to each region (for each bird) indicating the level of expression: $0=$ no labeled cells; $1=$ very few, lightly labeled cells; 2 = intermediate density of moderately stained cells; $3=$ high density of darkly labeled cells. This analysis was performed without knowledge of experimental treatment to replicate previous results showing that Fos is induced in RA and HVC by the act of singing (Kimpo and Doupe, 1997), and that non-singing birds did not express Fos-labeled cells in these regions.

The amount of song behavior produced by each Singing bird was calculated as: (1) the number of song bouts; (2) the total amount of vocal behavior produced, measured as the total number of $\mathrm{MB}$ for all files that included song production (files with calls only were eliminated). The quality of song behavior before versus after nerve lesions was assessed by examining printed sonograms and qualitatively evaluating the variety and morphology of individual syllables. At this age, most birds produce recognizable syllables, but do not always produce them in a consistent sequence. We therefore compared the similarity and quality of syllables pre- versus postlesion and assigned a "change score" for each bird according to the degree of change between the two time points (a span of 2-4 d): $0=$ no change, $1=$ slight change, $2=$ medium change, $3=$ substantial change. This analysis was conducted without knowledge of experimental treatment.

Nonparametric statistics were used to evaluate the data. The overall effect of groups (nerve section in Singing birds) was evaluated using a Kruskal-Wallis test. Non-singing birds were not included because, as described below, the induction of Fos expression in trigeminal regions and the solitary nucleus were too low to provide meaningful data in many birds. Comparisons between individual groups were evaluated using Mann-Whitney $U$ tests, and within-subject tests of neuronal density ipsilateral versus contralateral to nerve lesions were assessed using Wilcoxon signed-ranks tests.

\section{Results}

Qualitative examination of Fos labeling showed well labeled Fos nuclei throughout all levels of the brain in birds of all groups. Singing birds had high levels of Fos expression in the cortical nuclei RA and HVC, which control motor production of song (Kimpo and Doupe, 1997), whereas Non-singing birds showed little or no Fos expression in RA and HVC (cf. Jarvis and Nottebohm, 1997; Jin and Clayton, 1997). The median qualitative score for amount of Fos label in RA across all Singing birds was 3.0 (high density of darkly labeled cells; see Materials and Methods) compared with 0.0 in Non-singing birds (little or no expression) $(U=0, p=0.0001)$. The median qualitative score in HVC for Singing birds was also 3.0, compared with 1.0 for Non-singing birds (few, lightly labeled cells) $(U=0, p=0.0001)$. This pattern confirms that our behavioral manipulation was reflected in levels of Fos expression in the vocal motor pathway from HVC to RA. We observed low levels of Fos staining in the tracheosyringeal 
Table 1. Absolute density (number $/ \mathrm{mm}^{3}$ ) of Fos-labeled cells in all groups on intact (contralateral) versus lesioned (ipsilateral) side for each region (median \pm SEM)

\begin{tabular}{|c|c|c|c|}
\hline Experimental group & PrV & nTTD & $\mathrm{nTS}$ \\
\hline \multicolumn{4}{|l|}{ sXII + X, Singing } \\
\hline Intact & $6784 \pm 1969$ & $8579 \pm 1663$ & $13,206 \pm 4164$ \\
\hline Lesioned & $2795 \pm 474$ & $1891 \pm 1252$ & $9441 \pm 2880$ \\
\hline \multicolumn{4}{|l|}{$X$, Singing } \\
\hline Intact & $12,554 \pm 3956$ & $11,968 \pm 2236$ & $18,951 \pm 3129$ \\
\hline Lesioned & $11,820 \pm 4493$ & $10,520 \pm 1815$ & $11,814 \pm 2061$ \\
\hline \multicolumn{4}{|l|}{$\mathrm{XII}_{\mathrm{ts}}$, Singing } \\
\hline Intact & $8325 \pm 1069$ & $9691 \pm 4411$ & $11,902 \pm 6243$ \\
\hline Lesioned & $5786 \pm 932$ & $6196 \pm 4598$ & $11,304 \pm 6216$ \\
\hline \multicolumn{4}{|l|}{$\mathrm{XII}_{1}$, Singing } \\
\hline Intact & $23,694 \pm 8248$ & $22,961 \pm 5320$ & $23,005 \pm 3381$ \\
\hline Lesioned & $6620 \pm 2109$ & $10,382 \pm 1965$ & $25,351 \pm 4808$ \\
\hline \multicolumn{4}{|l|}{ No sxn, Singing } \\
\hline Intact & $3589 \pm 3912$ & $9039 \pm 2680$ & $14,573 \pm 5721$ \\
\hline Lesioned & $3634 \pm 3652$ & $5407 \pm 3553$ & $18,261 \pm 5629$ \\
\hline \multicolumn{4}{|l|}{ sXII + X, Non-singing } \\
\hline Intact & $1865 \pm 483$ & $1703 \pm 1373$ & $14,656 \pm 3900$ \\
\hline Lesioned & $1713 \pm 545$ & $2677 \pm 1643$ & $11,155 \pm 3675$ \\
\hline \multicolumn{4}{|l|}{ No Sxn, Non-singing } \\
\hline Intact & $168 \pm 109$ & $227 \pm 76$ & $12,766 \pm 1922$ \\
\hline Lesioned & $191 \pm 115$ & $0 \pm 34$ & $14,704 \pm 2315$ \\
\hline
\end{tabular}

hypoglossal motor nucleus $\left(\mathrm{nXII}_{\mathrm{ts}}\right.$ ) and in the dorsal $\mathrm{nX}$ of all Singing birds. The absence of staining in hypoglossal motor neurons is consistent with the results of Kimpo and Doupe (1997), and presumably is due to a lack of patterned neural activity that is appropriate for Fos induction in this area. We did not analyze these two motor regions further.

Our quantitative analysis of Fos labeling across all birds included regions of the descending and ascending trigeminal tracts that are known to receive afferents via the hypoglossal nerve, specifically nTTD and PrV (Wild, 1981, 1990; Bottjer and Arnold, 1982) (Fig. 1). In addition, we examined nTS, which receives afferent information from the vagus nerve.

\section{Principal sensory nucleus of the trigeminal tract}

Singing induces Fos expression in $\operatorname{PrV}$

We first examined the density of Fos-labeled cells in PrV across all Singing versus Non-singing birds on the intact control side (i.e., with no nerve lesion) to ask whether the act of song production induced Fos expression in this region (Table 1). Within $\operatorname{PrV}$, the mean density of labeled neurons on the intact side was $11,960 \pm 2223$ (mean \pm SEM) across all Singing birds compared with $1105 \pm 394$ in Non-singing birds ( $U=2, p=0.0003$ ). Thus, the act of singing was a highly effective stimulus for inducing Fos in PrV, suggesting that this region is part of the neural substrate for learned vocal behavior. We also examined the density of Fos-labeled cells on the intact control side as a function of the type of nerve section received in the five groups of Singing birds (including birds with no section; Table 1, Fig. 2). In general, birds in all five Singing groups showed increased expression of Fos (relative to Non-singing groups) and there were no significant differences between Singing groups, as expected $(H=7.75, p=0.10)$.

One important question is whether feeding behavior contributes to this pattern of Fos induction, since the lingual branch of the hypoglossal nerve, as well trigeminal axons from the beak and jaw, are known to convey afferents to sensory trigeminal regions (Wild, 1990). Although we did not monitor feeding behavior, birds typically eat at lights on. As described in Materials and

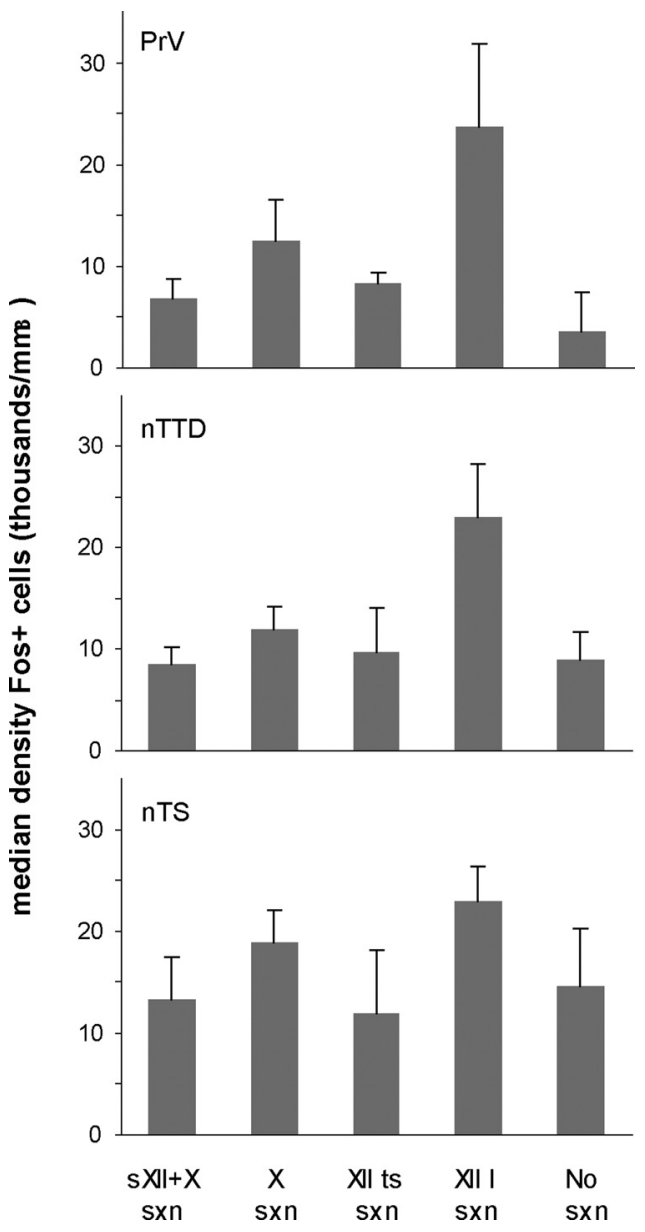

Figure 2. Median density ( + SEM) of Fos-positive cells $\left(\right.$ per $\mathrm{mm}^{3}$ ) in PrV, nTTD, and nTS on the intact control side (with no nerve lesion) in all Singing groups of birds.

Methods, non-singing control birds were removed from recording chambers shortly after lights on and placed in the main laboratory to prevent them from singing (before perfusion). These birds were observed for at least 15-20 min before being anesthetized, and would have been eating during that time. Because singing birds were left in the recording chambers for $0.5-2.0 \mathrm{~h}$ after lights on, they would have had more opportunities to eat. Nevertheless, if eating was an effective stimulus for inducing Fos expression in $\mathrm{PrV}$, then non-singing birds should have shown some induction. However, this was not the case: the absolute level of Fos expression was low in both groups of Non-singing birds (in PrV as well as nTTD; Table 1). Furthermore, one bird in the sXII + $\mathrm{X} /$ Non-singing group was observed for a full hour before being anesthetized; this bird showed a very low level of Fos expression in both PrV and nTTD (well below the range of all Singing birds) despite having the same amount of time to eat as most experimental birds. In contrast, the level of Fos induction in nTS was high in both Singing and Non-singing birds (see below and Table 1). Thus, it is highly unlikely that eating behavior contributed substantially to induction of Fos expression in PrV or nTTD, although we cannot rule out that some induction is due to feeding. This outcome could be because the pattern of neural activity induced by feeding is inappropriate to elicit Fos induction in trigeminal regions (similar to the of lack of singing-induced induction in $\left.\mathrm{nXII}_{\mathrm{ts}}\right)$. 


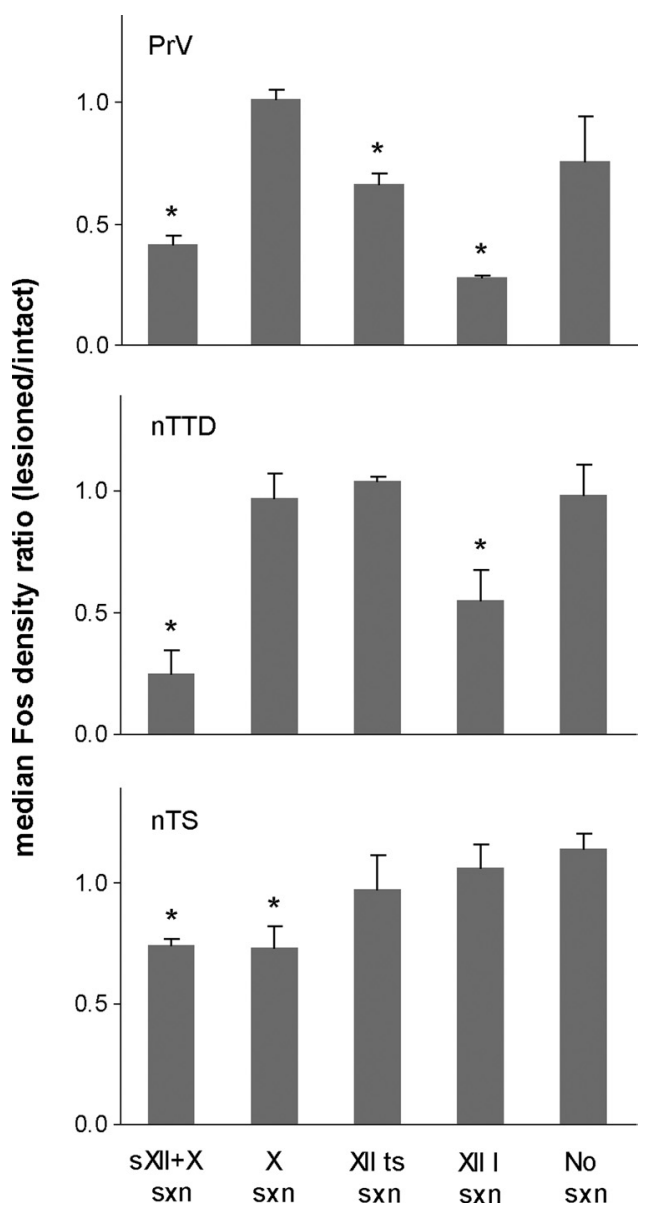

Figure 3. Median Fos density ratio ( + SEM) in all Singing groups of birds. Density ratios were calculated as the density of Fos + cells on the side with a nerve section divided by the density of Fos + cells on the intact side (in control birds with no nerve lesion this ratio was computed by dividing the density of Fos + cell on the left side by that on the right side). Ratio values of 1.0 indicate no difference between lesioned and intact sides; values $<1.0$ indicate decreased Fos expression on the side ipsilateral to the nerve section. * indicates significantly different from singing birds with no nerve section.

\section{Eliminating sensory hypoglossal afferents decreases Fos expression in $\operatorname{Pr} V$}

We next compared the level of Fos expression within each bird on the intact side to that on the lesioned side (i.e., the side with a unilateral nerve section) to test whether activity conveyed through sensory axons traveling in the main hypoglossal nerve influenced Fos induction. This difference was expressed as the ratio of Fos + neuronal density on the lesioned side divided by that on the intact side, such that ratio values of 1.0 indicate no difference between lesioned and intact sides, whereas lower values represent diminished Fos expression due to removal of sensory afferents (see Materials and Methods). The incidence of Fos + neurons in PrV was lower on the deafferented side in Singing birds that received a unilateral section of both sensory XII axons and the vagus (sXII $+\mathrm{X}$ section, Figs. $1 c, 3$; Table 1$)$. In contrast, Singing birds with section of X only (Fig. $1 d$ ) or with no nerve section showed no systematic difference between sides ipsiversus contralateral to the nerve lesion (this ratio was calculated as left/right for birds with no nerve section). Non-singing control birds showed no systematic difference in the density of Fos+ neurons on ipsi- versus contralateral sides regardless of whether they underwent section of XII afferents (sXII + X/Non-singing) or not (NoSxn/Non-singing) (Table 1). Thus, nerve section alone did not influence the pattern of Fos expression relative to the intact control side. Because the number of Fos-labeled cells in Non-singing control groups was very low in several birds, density ratios for these groups tended to be unreliable in the sense that very small changes in the absolute number of neurons between left versus right sides of the brain could yield spuriously large ratios. Hence, these data are not shown in Figure 3.

We were able to measure PrV in one Singing bird in which the sensory trunk of the main hypoglossal nerve was sectioned without damaging $\mathrm{X}$ axons (see Materials and Methods). The Fos density ratio for this bird was 0.41 , which fell in the middle of the range of birds with a nerve section of sXII $+\mathrm{X}$, and thus this bird was included in that group. This result agrees with the lack of an effect of vagal nerve section alone on Fos expression in PrV, and supports the idea that neural activity conveyed by hypoglossal afferents to PrV induces Fos expression. In summary, Fos expression was selectively decreased in PrV when sensory XII axons were removed, only in singing birds.

The sensory hypoglossal axons that we sectioned carry afferent information from both the syrinx and the tongue. To try to assess the contribution of syringeal versus lingual afferents, we sectioned these peripheral nerves separately. In these cases both sensory and motor axons (Fig. 1, red and gray, respectively) were sectioned in either the lingual or tracheosyringeal nerves. Birds with a section of either the lingual nerve $\left(\mathrm{XII}_{1}\right.$, Fig. $\left.1 a\right)$ or $\mathrm{nXII}_{\mathrm{ts}}$ (Fig. $1 b$ ) both showed a lower Fos density ratio (i.e., lower numbers of Fos-labeled nuclei in PrV on the lesioned side; Figs. 3, 4). This pattern of results shows that neural activity in both branches of the hypoglossal nerve convey sensory information from the tongue and syrinx during song production that induces Fos expression in PrV. In addition, it should be noted that the decreased Fos expression in PrV seen in singing birds with tracheosyringeal nerve lesions cannot be due to any contribution of eating behavior, since the syrinx plays no role in feeding.

A Kruskal-Wallis rank test on the Fos density ratios in PrV for all Singing groups demonstrated an overall effect of type of nerve section on ipsi- versus contralateral Fos + expression $(H=14.9, p=0.005)$. Individual group comparisons showed that Singing birds with section of either sXII $+\mathrm{X}, \mathrm{XII}_{\mathrm{ts}}$, or $\mathrm{XII}_{1}$ had lower Fos density ratios compared with Singing birds with no section ( $U=0, p<0.04$ in all cases). In addition, Fos density ratios in birds with section of $\mathrm{XII}+\mathrm{X}$ were lower than in birds with section of $\mathrm{X}$ only $(U=0, p=0.025)$. Last, despite tending toward a greater decrease in Fos expression on the sectioned side, birds in the $\mathrm{XII}_{1}$ group did not differ from those in the $\mathrm{XII}+\mathrm{X}$ group $(U=3, p=0.18)$.

Qualitative inspection of $\operatorname{PrV}$ indicated that it consisted of a dorsolateral and a ventromedial subdivision (Fig. 4) (Dubbeldam and Karten, 1978; Dubbeldam, 1980; Wild and Zeigler, 1996). The dorsolateral subdivision of PrV tended to contain more labeled cells than the ventromedial subdivision across all groups of Singing birds. In addition, birds with a section of sXII + X (or of sXII only) tended to have substantially more labeled cells in the lateral subdivision of $\mathrm{PrV}$ on the intact side compared with the sectioned side. That is, the difference in Fos expression between the sectioned and intact sides was more pronounced in the lateral subdivision of $\mathrm{PrV}$ compared with the medial subdivision. This pattern was also observed in birds with section of the lingual or tracheosyringeal nerves $\left(\mathrm{XII}_{1}\right.$ or $\mathrm{XII}_{\mathrm{ts}}$, respectively) but not in birds with section of $\mathrm{X}$ only or with no section. This pattern of results is consistent with the finding that the lateral subregion of $\mathrm{PrV}$ receives a high density of hypoglossal afferent fibers (Bottjer and Arnold, 1982; Wild, 1990). 
The main efferent projection of $\mathrm{PrV}$ in pigeons bypasses the thalamus and travels directly to the telencephalon, terminating in the basorostral nucleus (Bas) of the frontal nidopallium (NF; Wild et al., 1985). Because projections from PrV to Bas are strongly bilateral, we did not expect to see differences in singing-induced gene expression ipsi- versus contralateral to nerve lesions. This was true, for the somewhat surprising reason that Bas was unlabeled in singing birds for either Fos or ZENK (see Materials and Methods). This pattern suggests either that the pattern of neural activity induced by singing is ineffective in inducing IEG expression in Bas, and/or that the Fos + cells in PrV of songbirds have a separate efferent target.

\section{Nucleus of the descending trigeminal tract}

We identified a distinct cell group within the nucleus of the descending trigeminal tract. This nucleus lies just ventral to the axons of the VIIIth nerve at the level of the medial vestibular nucleus and the magnocellular nucleus of the cochlea, just rostral to the level of the dorsal motor nucleus of the vagus (Fig. 4, arrow). We refer to this nucleus as nTTD (as a generic name); it lies within the "pars interpolaris" region of the nucleus of TTD. Previous studies observed that this region is composed of several cell groups in pigeons and mallards (Dubbeldam and Karten, 1978; Dubbeldam, 1980; Arends and Dubbeldam, 1984; Wild and Zeigler, 1996), which they did not attempt to differentiate (but see below).

\section{Singing induces Fos expression in nTTD}

As in PrV, the act of singing was highly effective for inducing Fos expression in nTTD. The mean density of Fos + neurons in nTTD on the intact side was $11,455 \pm 1518$ across all Singing birds compared with $1397 \pm 780$ in Non-singing birds $(U=8, p=$ 0.0002; Table 1). The induction of Fos by singing in both nTTD and $\operatorname{PrV}$ indicates that neural activity is upregulated in both the descending and ascending trigeminal tracts during vocal production as a result of sensory feedback from vocal articulators. As in $\mathrm{PrV}$, the density of Fos-labeled cells in nTTD did not vary across different groups of Singing birds as a function of type of nerve section $(H=4.44, p=0.35$; Fig. 2$)$.

Eliminating sensory hypoglossal afferents decreases Fos expression in $n T T D$

Singing birds with a section of sensory XII axons and the vagus (sXII + X; Fig. 1c) had a low number of labeled Fos nuclei in nTTD on the side ipsilateral to the lesion relative to the intact contralateral side (Figs. 3, 4). In contrast, Singing birds with no section or with a unilateral section of the vagus (X only; Fig. 1d) showed equal levels of Fos expression ipsi- and contralateral to the lesion. Non-singing control birds showed no systematic difference in the density of Fos + neurons on ipsi- versus contralateral sides regardless of whether they did or did not have a section of XII afferents (sXII + X/Non-singing and NoSxn/Non-singing, respectively; Table 1). Thus, as in the case of $\operatorname{PrV}$, either no nerve section at all or section of the vagus alone had no effect on Fos expression in Singing birds. In addition, although eliminating sensory hypoglossal afferents by sectioning sXII + X did not influence the pattern of Fos expression in Non-singing birds, the same lesion prevented the induction of Fos in Singing birds.

We were able to measure nTTD in two Singing birds in which the sensory axons of the main hypoglossal nerve were sectioned without damaging X axons (see Materials and Methods). The Fos density ratios for both of these birds ( 0.13 and 0.30 , respectively) fell within the range of values for birds with a unilateral section of sXII $+\mathrm{X}$, and were thus included with this latter group. As in the case of $\mathrm{PrV}$, this pattern is consistent with the idea that decreased Fos expression in nTTD is due to elimination of hypoglossal sensory afferents, and is not influenced by removing sensory inputs from the vagus.

Comparison of birds that received a unilateral section of either the lingual or the tracheosyringeal hypoglossal nerve (including both sensory and motor axons; Fig. $1 a, b$ ) revealed no difference in nTTD as a result of lesioning the tracheosyringeal nerve (Fig. 3). However, sectioning the lingual nerve decreased the incidence of Fos-labeled cells in nTTD on the deafferented side. This pattern indicates that activity in lingual afferents during song production enhances the expression of Fos in nTTD. In this case, section of sXII plus X axons produced the strongest effect, which no combination of other nerve sections seemed to fully reproduce. That is, section of the vagus or of nXII $_{t s}$ alone did not exert any effect, and section of the lingual nerve appeared to exert less of an effect compared with section of sXII + X (although this difference was not significant; see below).

A Kruskal-Wallis test on Fos density ratios in nTTD for all Singing groups revealed an overall effect of nerve section $(H=$ 
$14.2, p=0.007)$. Individual comparisons showed that Fos density ratios in both $\mathrm{XII}+\mathrm{X}$ and $\mathrm{XII}_{1}$ groups were different from those in birds with no section $(U=3, p=0.02$, and $U=2, p=$ 0.05 , respectively), whereas birds with a section of $\mathrm{XII}_{\mathrm{ts}}$ were not different from birds with no section $(p=0.66)$. Fos density ratios were lower in birds with a section of sXII $+X$ than in birds with section of $\mathrm{X}$ only $(U=0, p=0.008)$, and birds in the $\mathrm{XII}+\mathrm{X}$ group were not different from birds with a section of $\mathrm{XII}_{1}(p=0.13)$.

Post hoc inspection of nTTD revealed a region just dorsolateral to the area we measured (Fig. 4, arrowhead). Qualitative inspection showed that Singing birds with a section of sXII + X showed a pronounced decrease in Fos expression within this dorsolateral region on the side with the nerve section. This region of nTTD had many more Fos-labeled cells on the intact side compared with the lesioned side in all seven birds that received a unilateral section of sXII + X. All four birds that received a section of the lingual branch of the hypoglossal nerve $\left(\mathrm{XII}_{1}\right)$ also showed a pronounced decrease in Fos induction in this area of nTTD on the lesioned side (Fig. 4). In contrast, birds with a section of the $\mathrm{nXII}_{\mathrm{ts}}$ showed no difference in this region between intact and lesioned sides, and also exhibited low absolute levels of Fos induction in this area. Birds with a section of the vagus also showed no difference between lesioned and intact sides in this region. Although we had not identified these two cell groups within nTTD previously, in retrospect they can be clearly identified even in Nissl sections, and may represent medial and lateral subdivisions of a single nucleus. In addition, the dorsolateral cell group seems to correspond to a subregion within the pars interpolaris region of TTD that receives a high density of afferent fibers via the main sensory hypoglossal trunk, including lingual inputs; it is not known whether this region receives tracheosyringeal inputs (Wild, 1981, 1990; Bottjer and Arnold, 1982).

\section{Nucleus of the solitary tract}

Singing does not induce Fos expression in nTS

We compared the density of labeled cells in nTS across all Singing versus Non-singing birds on the intact side to test whether song production induced Fos expression in this region (Table 1). The mean density of Fos-labeled neurons in nTS on the intact side was $17,920 \pm 9270$ across all Singing birds compared with 13,229 \pm 5877 in Non-singing birds $(U=72.0, p=0.24)$. Thus, the act of singing was not an effective stimulus for inducing Fos in nTS. In addition, absolute densities of Fos-labeled cells on the intact side of nTS did not vary as a function of experimental treatment across Singing birds $(H=1.60, p=0.81$; Fig. 2, Table 1$)$.

\section{Eliminating afferents from the vagus decreases Fos expression} in $n T S$

Singing birds with a section of either sXII + X or of X only (Fig. $1 c, d$, respectively) showed a low number of labeled Fos nuclei on the side ipsilateral to the lesion relative to the intact (contralateral) side (Figs. 3, 4; Table 1). In contrast, Singing birds with a section of either the lingual or the tracheosyringeal hypoglossal nerve (including both sensory and motor axons) showed no difference in Fos expression in nTS ipsi- versus contralateral to the nerve section. Singing birds with no nerve section also showed no systematic difference in Fos expression between left versus right sides of nTS. In addition, Non-singing birds with either no section or a unilateral section of sXII + X showed no difference (Table 1). This pattern of results indicates that Fos expression in nTS was diminished only by section of the vagus in Singing birds. Thus, despite the absence of an overall difference in Fos expres- sion in nTS between Singing and Non-singing birds, these results suggest that sensory X afferents terminating in nTS are specifically recruited during singing.

A Kruskal-Wallis test on Fos density ratios in nTS revealed a main effect of groups for Singing birds $(H=13.5, p=0.01)$. Individual group comparisons revealed that singing birds with a section of either sXII + X or X alone had lower Fos density ratios than birds with no section ( $U=0, p=0.01$ in both cases). These two groups did not differ from each other $(p=0.81)$. Fos density ratios of birds with a unilateral section of either $\mathrm{XII}_{\mathrm{ts}}$ or $\mathrm{XII}_{1}$ were not different from those of control birds with no section $(U=4$, $p=0.14$, and $U=8, p=0.62$, respectively). Thus, birds that received a unilateral nerve section of sXII + X showed a reduction in Fos labeling similar to those that received a section of $\mathrm{X}$ only, indicating that lesion of hypoglossal sensory afferents from the tongue and syrinx do not influence Fos expression in the solitary nucleus. This pattern suggests that afferent information conveyed via the vagus during vocal production is the primary determinant of neural activity and hence Fos expression in the solitary nucleus.

As an additional test of whether sensory vagal afferents are recruited during song production, we compared Fos density ratios between Singing birds with a section of sXII + X or X only versus Non-singing birds with a section of sXII $+\mathrm{X}$; this comparison was not significant $(U=8, p=0.12)$. This lack of a significant difference is likely due to the tendency toward higher Fos densities in nTS on the intact side in Non-singing birds with a section of sXII + X (Table 1$)$, which was primarily due to one bird in this group that had a much higher density of Fos + cells on the intact side. In accord with this idea, Wilcoxon signed-ranks tests on Fos densities for the intact versus lesioned sides showed a significant effect only in Singing birds with a section of sXII $+\mathrm{X}$ or of X only $(p<0.05)$ but not in either group of Non-singing birds $(p>0.40)$. In addition, density ratios in Singing birds with a section of either $\mathrm{XII}+\mathrm{X}$ or of X alone were lower than those in Non-singing birds with no section $(U=0, p=0.003)$. Thus these results are consistent with the idea that section of the vagus diminishes Fos expression that is otherwise induced in nTS of singing birds.

\section{Singing behavior}

Qualitative examination of preoperative song behavior showed that approximately half of all birds had developed a stable sequence of syllables by this stage of vocal development, which corresponds to mid-stages of sensorimotor integration. Despite the fact that many birds lacked a stereotyped temporal sequence, the majority produced syllables that were easily recognizable (only one bird produced vocalizations that were sufficiently immature that they could not be consistently recognized as individual syllables). We assigned a "change score" from 0 (no change) to 3 (substantial change) to assess the degree of alteration in vocal behavior following a nerve lesion (see Materials and Methods). This analysis revealed that only birds with a section of the tracheosyringeal nerve showed substantial alterations in song. The median change score for birds in this group was 2.75, whereas birds with section of the lingual nerve, the vagus, or sXII + X had median change scores of $0.0,1.0$, and 1.0, respectively. Two birds underwent section of the right $\mathrm{XII}_{\mathrm{ts}}$ nerve, and showed highly disrupted song behavior (change score $=3$ in both cases), whereas two other birds underwent a section of the left XII nerve, and showed somewhat less substantive disruption of behavior (change scores $=2.0-2.5$ ). Figure 5 shows examples of song behavior from a bird before and after a lesion of the right 
tracheosyringeal nerve. This bird produced normal plastic song consisting of recognizable syllables with little or no stable sequence before the nerve section. Two days following the lesion this bird produced a song consisting entirely of simple low-amplitude syllables that tended to be noisy and poorly modulated. In addition, none of the postoperative syllables could be matched to any preoperative syllables, and the long calls produced by this bird showed little or no frequency modulation. This pattern of results confirms previous studies indicating that lesion of the tracheosyringeal nerve, particularly of the right nerve, produces gross disruption of vocal behavior in zebra finches (Williams et al., 1992).

It has been suggested that because the tongue of birds is not highly deformable, it may not play a major role in vocal production; in addition, lingual motor neurons receive no direct cortical projection as the tracheosyringeal motor neurons do (Wild, 1993a, 2004). Only one of four birds showed some noticeable change in song following unilateral section of the lingual nerve. To further test whether the tongue contributes to vocal production, we lesioned the lingual nerve bilaterally in two additional birds and recorded their vocal behavior for 1 week. These birds showed no obvious disruption in either song behavior (data not shown) or feeding behavior, as evidenced by the fact that neither bird showed any weight loss 1 week following bilateral section of the lingual nerve. One possible reason that lingual nerve sections do not exert a large effect is that innervation of the tongue by the glossopharyngeal and facial nerves also contributes to both feeding and singing and/or that extrinsic tongue muscles and the hyoid apparatus are necessary for feeding and singing (Wild, 1990). Extrinsic tongue muscles and the hyoid apparatus are innervated by the facial and glossopharyngeal nerves in pigeons and ducks (Dubbeldam et al., 1979; Wild and Zeigler, 1980; Dubbeldam, 1984), and innervation of the beak and jaw by the trigeminal nerve is necessary for normal ingestive pecking (Bermejo and Zeigler, 1989, 1999), so it is likely that other cranial nerves contribute to both normal song production and to eating behavior. Thus, lesions of the lingual nerve alone may be insufficient to unmask a contribution of the tongue to vocal behavior.

Although birds with a unilateral section of the vagus did not show substantive disruption of vocal behavior as a group, two of these four birds received change scores of 2.0 and 2.5, respectively, suggesting that vagal lesions can influence song. This result is very similar to that reported by Méndez et al. (2010), who reported that unilateral vagotomy of adult zebra finches caused alterations in the normal respiratory pattern during song, sometimes leading to acoustic changes such as truncations and interruptions. These disruptions occurred within the first few days postsurgery, followed by partial or full recovery, and were highly variable between individual birds, as observed here. Although we did not measure respiratory patterns directly, as did Méndez et al. (2010), the effects we observed in juvenile birds were consistent with this pattern. In addition, both of the birds that showed noticeable changes in song tended to produce slightly noisier and more variable syllables, suggesting a decreased ability to modulate air flow and pressure through the syrinx. Although these behavioral effects are considerably less dramatic than those induced by tracheosyringeal nerve lesions, this difference could be at least partly attributable to the fact that innervation of the syrinx by the tracheosyringeal nerve seems to be highly lateralized, whereas the respiratory motor program for song appears to be symmetrical (Goller and Cooper, 2004; Suthers and Zollinger, 2004).

We quantified the amount of song produced by each bird during the morning before the time the bird was perfused both by counting the number of song bouts produced and by estimating the total amount of song produced as the total number of MB of all files that included song behavior (see Materials and Methods). We compared the amount of singing to the density of Fos-labeled cells on the intact side to test for a positive correlation. Birds that sang more tended to have higher levels of Fos-labeled cells in nTTD. The correlation between the number of song bouts and the density of Fos + cells in nTTD was not significant $(r=0.33$, Pearson correlation coefficient; $p=0.17$ ) but the correlation between total MB of song and density of Fos + cells in nTTD was marginally significant $(r=0.45, p=0.06)$. In contrast, correlations between amount of singing and Fos + density in PrV did not approach significance $(F<1)$. This pattern is in contrast to studies showing that the level of IEG expression in RA and HVC is significantly correlated with amount of singing (Jin and Clayton, 1997; Kimpo and Doupe, 1997; Jarvis et al., 1998). It is possible that Fos expression in the brainstem differs from cortex in that it has an all or none response to singing.

In summary, the behavioral results of tracheosyringeal and vagal nerve lesions are consistent with the ability of nerve sections to decrease singing-induced Fos expression, in the sense that each nerve contributes to vocal production. Although lingual nerve sections did not exert a behavioral effect, our results do not rule 


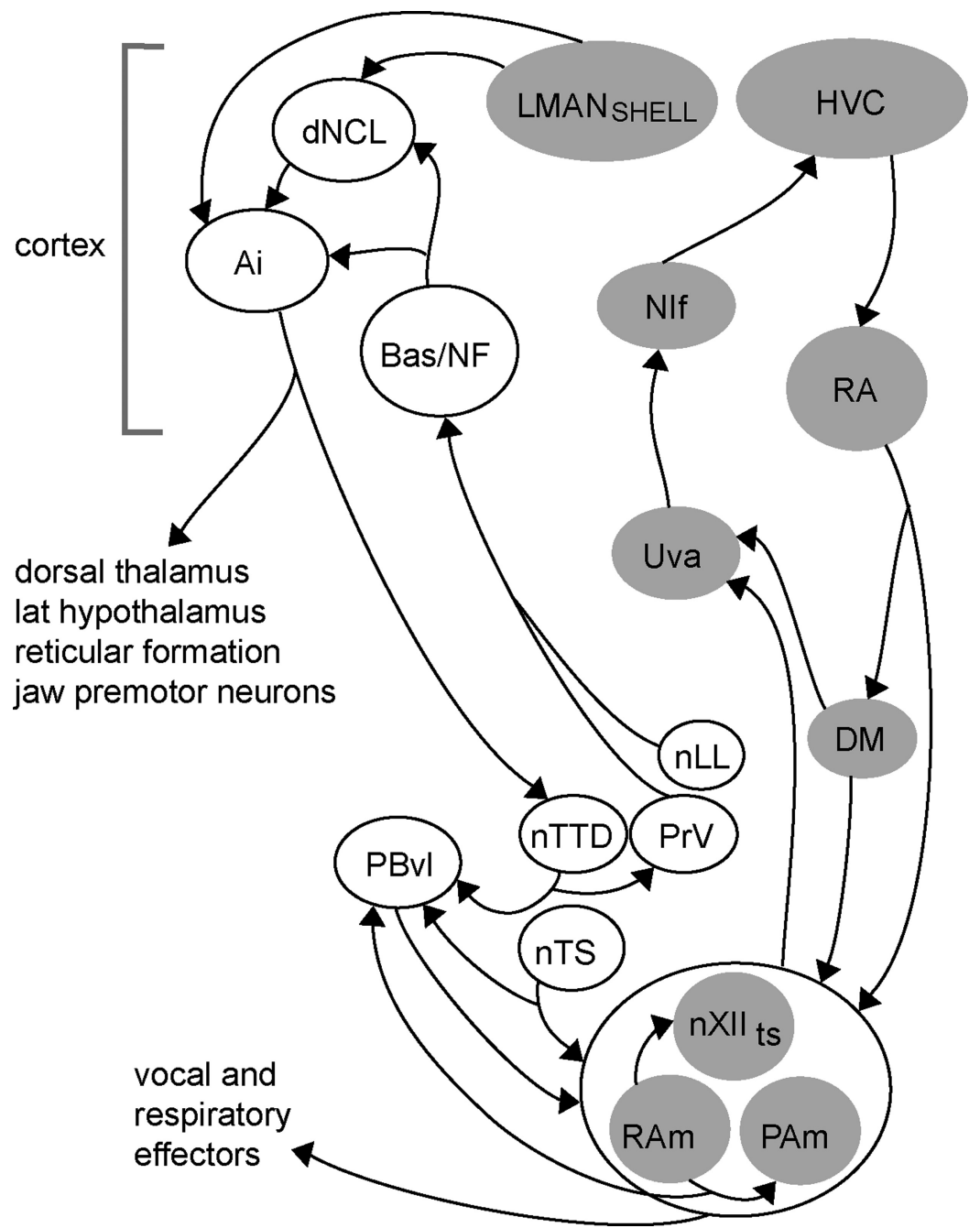

Figure 6. Schematic diagram of brain regions involved with vocal control and learning (gray), and brain regions involved with respiratory and somatosensory information processing (white). Many connections have been omitted for the sake of clarity Sensory hypoglossal and vagal information conveyed to PRv, nTTD, and nTS have numerous points of interconnection with vocal-control circuits. In particular, projections from trigeminal and auditory regions converge in $\mathrm{NNCL}$ and Ai with projections from $\mathrm{LMAN}_{\text {shelli }}$ this latter pathway is necessary for imitative vocal learning in juvenile zebra finches (Bottjer and Altenau, 2009). Thus, Ai is a song-control region in zebra finches, but it is not known whether the same region of Ai receives inputs from both $L_{M A N}$ shell and auditory/trigeminal regions; hence both Ai and dNCL are shown in white here; see Discussion. Based on previous work (Wild, 1981, 1990, 1993a,b; Bottjer and Arnold, 1982; Arends et al., 1984, 1988; Wild et al., 1985, 1990, 1997; Wild and Farabaugh, 1996; Reinke and Wild, 1997, 1998; Striedter and Vu, 1998; Sturdy et al., 2003; Ashmore et al., 2008; Wild and Krützfeldt, 2012). DM, Dorsomedial nucleus of the inferior colliculus; $\mathrm{LMAN}_{\text {shell, }}$, shell region of the lateral magnocellular nucleus of the anterior nidopal-

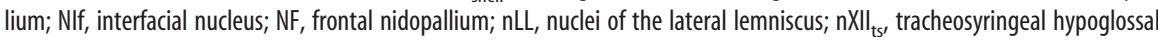
nucleus; PBvl, ventrolateral portion of the parabrachial nucleus; PAm, nucleus parambigualis; PBvl, ventrolateral parabrachial nucleus; RAm, nucleus retroambigualis; Uva, uvaeform nucleus of the thalamus.

out a contribution of the tongue in song production. The lack of an effect is likely due to the fact that both facial and glossopharyngeal nerves also contribute to movement of the tongue. Of course, all of these sections involved both motor and sensory axons, and so do not test a specific role for afferent inputs, but do testify to a basic involvement of the tracheosyringeal and vagal nerves in control of vocalizations.

\section{Discussion}

We sectioned the sensory axons of the hypoglossal nerve unilaterally in juvenile songbirds during the sensorimotor period of vocal learning and then compared the level of singing-induced Fos expression on the intact side of the brainstem to that on the deafferented side within each bird. Our prediction was that if sensory inputs from vocal effectors are active during vocal production, then singing birds should show induction of Fos on the intact side and a decreased level on the lesioned side. This prediction was confirmed: Fos expression was induced in nuclei of the trigeminal tract on the intact side only in singing birds and not in nonsinging birds. Furthermore, eliminating sensory axons in the hypoglossal nerve substantially diminished singing-induced Fos expression. In addition, singing birds with a section of hypoglossal afferents showed no difference in ipsi- versus contralateral regions of the solitary tract. This pattern suggests that sensory afferents from the tongue and syrinx to trigeminal regions convey information related to vocal behavior in juvenile birds during sensorimotor integration, such that both auditory and somatosensory inputs contribute to the refinement of vocal motor patterns as birds are learning to match the memory of tutor song.

In addition, we made unilateral sections of either the lingual or the tracheosyringeal branch of the hypoglossal nerve to assess the differential contribution of afferents from the tongue versus syrinx. Although this manipulation lesions both efferent and afferent axons, it should effectively eliminate sensory inputs on that side. The resulting pattern of Fos expression showed that both tracheosyringeal and lingual sensory fibers contribute to Fos expression in PrV of singing birds. Only lingual afferents contributed to Fos expression in the regions within nTTD that we examined, although we cannot rule out that tracheosyringeal afferents induce Fos expression in other regions of nTTD or elsewhere.

Singing birds with a unilateral section of the vagus showed decreased Fos expression in nTS ipsilateral to the nerve section, but no systematic difference in trigeminal regions. Section of the vagus caused a greater reduction of Fos expression on the lesioned side of nTS in singing birds than in non-singing birds, suggesting that respiratory information specific to singing is conveyed to nTS. Singing-induced Fos expression in nTS was not particularly robust, and may have been partially obscured by the high overall levels of Fos expression in nTS of both singing and non-singing birds (Table 1); possibly normal respiration in nonsinging birds also contributes to Fos expression in nTS.

\section{Functional circuits for song control in relation to vocal motor feedback}

How might hypoglossal sensory inputs to trigeminal regions interface with circuits that contribute to learning and production of vocal behavior? Trigeminal brainstem regions are potentially key loci for coordinating various sensory inputs with vocal effectors, 
since they also receive afferent inputs from upper vocal tract structures such as the beak, jaw, and oral cavity (Wild et al., 1984; Arends and Zeigler, 1986; Wild and Farabaugh, 1996; Wild, 2004, 2008; Wild and Krützfeldt, 2012). One possible route between trigeminal regions and circuits for song-control involves a projection from PrV and nuclei of the lateral lemniscus (nLL) to telencephalic somatosensory/auditory regions (Bas and NF) that project in turn to regions of dorsal caudo-lateral cortex (dNCL) and motor cortex (intermediate arcopallium, Ai; Fig. 6). Although this circuit was originally discovered in pigeons (which do not learn their vocalizations), much of it has also been demonstrated in zebra finches (Wild et al., 1985; Wild and Farabaugh, 1996; Wild and Krützfeldt, 2012). Furthermore, both Ai and $\mathrm{dNCL}$ in zebra finches are part of a circuit including a subregion of the song-control nucleus LMAN (shell region of the lateral magnocellular nucleus of the anterior nidopallium), which is essential for vocal learning in juvenile birds (Bottjer et al., 2000; Bottjer and Altenau, 2010). In addition, Ai makes projections to downstream regions including nTTD, lateral hypothalamus, dorsal thalamus, reticular formation, and jaw premotor neurons located adjacent to vocal motor and pre-respiratory neurons $\left(\mathrm{nXII}_{\mathrm{ts}}, \mathrm{RAm}\right.$, and PAm; Fig. 6). This pattern of connectivity suggests that both auditory (nLL) and somatosensory (PrV, nTTD) information may be conveyed to cortical song-control regions (such as $\mathrm{dNCL}$ ), which then complete recurrent connections back to nTTD via Ai.

One caveat is that the extent to which the termination zones of this auditory-somatosensory loop overlap directly with songcontrol circuits within dNCL and Ai is currently unknown, although they appear to have several points of convergence which suggest multiple opportunities for integration between somatosensory, auditory, and vocal-control pathways (cf. Wild and Farabaugh, 1996; Bottjer et al., 2000). For example, projections from $\mathrm{LMAN}_{\text {shell }}$ and NF (Fig. 6) may converge in dNCL, an area that is similar to a cortical region necessary for imprinting in chicks (Metzger et al., 1998; Braun et al., 1999). dNCL projects in turn to Ai, which connects back to the dorsal thalamus (and thence to LMAN and indirectly to HVC), to the trigeminal complex, and to other shared targets (such as reticular formation and lateral hypothalamus). In addition, nTTD in mallards projects to both PrV and PBvl (ventrolateral parabrachial nucleus; Arends et al., 1984); PBvl is involved with regulation of respiratory phase and projects to lateral hypothalamus, dorsal thalamic nuclei, striatum, and arcopallium, as well as to nXIIts and adjacent premotor respiratory neurons (Fig. 6; Wild et al., 1990). In addition, nTTD makes a projection to a thalamic nucleus in mallards that may correspond to the dorsal thalamic region in songbirds that projects to LMAN and HVC. If confirmed, such a projection would link hypoglossal and trigeminal afferents directly into circuitry essential for vocal learning.

Another important interface by which feedback from peripheral receptors is interfaced with song-control regions may be provided by the solitary complex. Syringeal afferents have been reported to terminate in nTS as well as in trigeminal regions, and nTS neurons that receive pulmonary afferents project heavily to $\mathrm{PBvl}$, which projects in turn to the vocalrespiratory efferent complex $\left(\mathrm{nXII}_{\mathrm{ts}} / \mathrm{RAm} / \mathrm{PAm}\right)$, thereby demonstrating a direct connection between respiratory and vocal systems (Wild et al., 1984, 1990; Wild and Arends, 1987; Wild, 2004). In addition, inspiratory premotor neurons (PAm) project to the thalamic nucleus Uva (uvaeform nucleus of the thalamus; Wild, 1994), which projects in turn to the auditory-motor song-control regions NIf (interfacial nucleus) and HVC (Fig. 6). The avian respiratory system includes air sacs containing receptors innervated by the vagus (Kubke et al., 2004), and artificially increasing respiratory pressure during singing (but not during quiet respiration) elicits an immediate compensatory reduction in activity of abdominal expiratory muscles (Suthers et al., 2002). These findings suggest nTS and its connections to vocal-respiratory brainstem regions as important loci in functional circuits for vocal control. For example, the projections of both nTTD and nTS to $\mathrm{PBvl}$ could be fed forward to Uva via the $\mathrm{nXII}_{\mathrm{ts}} / \mathrm{RAm} / \mathrm{PAm}$ complex.

It is likely that many of the connections linking sensory trigeminal and vagal regions to song-control circuitry have yet to be discovered. In addition, it is undoubtedly true that the known plexus of connections between both sensory and motor components of various cranial nerves innervating vocal effectors (including V, VII, IX, X, and XII) at the level of the pons and medulla represents only a subset of the possible functional connections that subserve vocal behavior. Thus, it is possible that sensory afferents from diverse peripheral effectors contribute to coordination of vocal and respiratory systems via interactions throughout the neuraxis.

\section{Contribution of peripheral effectors to vocal behavior}

The behavioral results of sectioning various nerves showed, as reported previously, that unilateral section of the tracheosyringeal nerve causes profound disruption of vocal behavior, and also reduces Fos expression in PrV of singing birds ipsilateral to the lesion. No published reports have determined whether $\mathrm{XII}_{\mathrm{ts}}$ afferents send a terminal projection to PrV, although unpublished work from our lab suggests that is true. At first glance, the fact that unilateral section of the lingual and vagal nerves did not cause profound disruption of vocal behavior may seem at odds with the pattern of Fos induction showing that singing-induced neural activity is conveyed via the lingual nerve to PrV and nTTD, and that singing also appears to induce Fos expression in nTS via the vagal nerve. However, as described above, the lack of strong effects may be more attributable to the fact that multiple cranial nerves are important for controlling the tongue, and that bilateral control of respiration may also contribute to vocal production.

In summary, these results provide functional evidence for a role of somatosensory afferents from the tongue and syrinx to brainstem trigeminal regions and of vagal afferents to the solitary complex in vocal behavior of juvenile zebra finches that are actively engaged in learning. Hypoglossal nerve sections did not produce a decrease in Fos expression in nTS, and vagal nerve sections had no effect on Fos expression in trigeminal regions. This specificity suggests the possibility that vocal motor and respiratory feedback enter the brainstem via separate routes and are integrated at subsequent points of interface.

\section{References}

Arends JJ, Dubbeldam JL (1984) The subnuclei and primary afferents of the descending trigeminal system in the mallard (Anas platyrhynchos L.). Neuroscience 13:781-795.

Arends JJ, Zeigler HP (1986) Anatomical identification of an auditory pathway from a nucleus of the lateral lemniscal system to the frontal telencephalon (nucleus basalis) of the pigeon. Brain Res 398:375-381.

Arends JJ, Woelders-Blok A, Dubbeldam JL (1984) The efferent connections of the nuclei of the descending trigeminal tract in the mallard (Anas platyrhynchos L.). Neuroscience 13:797-817.

Arends JJ, Wild JM, Zeigler HP (1988) Projections of the nucleus of the tractus solitarius in the pigeon (Columba livia). J Comp Neurol 278:405-429. 
Ashmore RC, Renk JA, Schmidt MF (2008) Bottom-up activation of the vocal motor forebrain by the respiratory brainstem. J Neurosci 28:2613-2623.

Baptista LF, Morton ML (1988) Song learning in montane white-crowned sparrows-from whom and when. Anim Behav 36:1753-1764.

Beckers GJ, Nelson BS, Suthers RA (2004) Vocal-tract filtering by lingual articulation in a parrot. Curr Biol 14:1592-1597.

Bermejo R, Zeigler HP (1989) Trigeminal deafferentation and prehension in the pigeon. Behav Brain Res 35:55-61.

Bermejo R, Zeigler HP (1999) Trigeminal deafferentation and conditioned pecking in pigeons. Behav Brain Res 99:181-189.

Bottjer SW, Altenau B (2010) Parallel pathways for vocal learning in basal ganglia of songbirds. Nat Neurosci 13:153-155.

Bottjer SW, Arnold AP (1982) Afferent neurons in the hypoglossal nerve of the zebra finch (Poephila guttata): localization with horseradish peroxidase. J Comp Neurol 210:190-197.

Bottjer SW, Arnold AP (1984) The role of feedback from the vocal organ. I. Maintenance of stereotypical vocalizations by adult zebra finches. J Neurosci 4:2387-2396.

Bottjer SW, Brady JD, Cribbs B (2000) Connections of a motor cortical region in zebra finches: relation to pathways for vocal learning. J Comp Neurol 420:244-260.

Braun K, Bock J, Metzger M, Jiang S, Schnabel R (1999) The dorsocaudal neostriatum of the domestic chick: a structure serving higher associative functions. Behav Brain Res 98:211-218.

Dubbeldam JL (1980) Studies on the somatotopy of the trigeminal system in the mallard, Anas platyrhynchos L. II. Morphology of the principal sensory nucleus. J Comp Neurol 191:557-571.

Dubbeldam JL (1984) Afferent connections of nervus facialis and nervus glossopharyngeus in the pigeon (Columba livia) and their role in feeding behavior. Brain Behav Evol 24:47-57.

Dubbeldam JL, Karten HJ (1978) The trigeminal system in the pigeon (Columba livia). I. Projections of the gasserian ganglion. J Comp Neurol 180:661-678.

Dubbeldam JL, Brus ER, Menken SB, Zeilstra S (1979) The central projections of the glossopharyngeal and vagus ganglia in the mallard, Anas platyrhynchos L. J Comp Neurol 183:149-168.

Feng Y, Gracco VL, Max L (2011) Integration of auditory and somatosensory error signals in the neural control of speech movements. J Neurophysiol 106:667-679.

Fletcher NH, Riede T, Suthers RA (2006) Model for vocalization by a bird with distensible vocal cavity and open beak. J Acoust Soc Am 119:1005-1011.

Goller F, Cooper BG (2004) Peripheral motor dynamics of song production in the zebra finch. Ann N Y Acad Sci 1016:130-152.

Ito T, Tiede M, Ostry DJ (2009) Somatosensory function in speech perception. Proc Natl Acad Sci U S A 106:1245-1248.

Jarvis ED, Nottebohm F (1997) Motor-driven gene expression. Proc Natl Acad Sci U S A 94:4097-4102.

Jarvis ED, Scharff C, Grossman MR, Ramos JA, Nottebohm F (1998) For whom the bird sings: context-dependent gene expression. Neuron 21:775-788.

Jin H, Clayton DF (1997) Localized changes in immediate-early gene regulation during sensory and motor learning in zebra finches. Neuron 19:1049-1059.

Johnson F, Soderstrom K, Whitney O (2002) Quantifying song bout production during zebra finch sensory-motor learning suggests a sensitive period for vocal practice. Behav Brain Res 131:57-65.

Kimpo RR, Doupe AJ (1997) FOS is induced by singing in distinct neuronal populations in a motor network. Neuron 18:315-325.

Kubke MF, Ross JM, Wild JM (2004) Vagal innervation of the air sacs in a songbird, Taenopygia guttata. J Anat 204:283-292.

Kuhl PK (2004) Early language acquisition: cracking the speech code. Nat Rev Neurosci 5:831-843.

Mello CV, Ribeiro S (1998) ZENK protein regulation by song in the brain of songbirds. J Comp Neurol 393:426-438.

Méndez JM, Dall'asén AG, Goller F (2010) Disrupting vagal feedback affects birdsong motor control. J Exp Biol 213:4193-4204.

Metzger M, Jiang S, Braun K (1998) Organization of the dorsocaudal neostriatal complex: a retrograde and anterograde tracing study in the domestic chick with special emphasis on pathways relevant to imprinting. J Comp Neurol 395:380-404.
Mongeau R, Miller GA, Chiang E, Anderson DJ (2003) Neural correlates of competing fear behaviors evoked by an innately aversive stimulus. J Neurosci 23:3855-3868.

Morrison RG, Nottebohm F (1993) Role of a telencephalic nucleus in the delayed song learning of socially isolated zebra finches. J Neurobiol 24:1045-1064.

Nasir SM, Ostry DJ (2006) Somatosensory precision in speech production. Curr Biol 16:1918-1923.

Nasir SM, Ostry DJ (2008) Speech motor learning in profoundly deaf adults. Nat Neurosci 11:1217-1222.

Nasir SM, Ostry DJ (2009) Auditory plasticity and speech motor learning. Proc Natl Acad Sci U S A 106:20470-20475.

Nottebohm F (1971) Neural lateralization of vocal control in a passerine bird. 1. Song. J Exp Zool 177:229-261.

Nottebohm F, Nottebohm ME (1976) Left hypoglossal dominance in control of canary and white-crowned sparrow song. J Comp Physiol A Neuroethol Sens Neural Behav Physiol 108:171-192.

Nottebohm F, Manning E, Nottebohm ME (1979) Reversal of hypoglossal dominance in canaries following unilateral syringeal denervation. J Comp Physiol A Neuroethol Sens Neural Behav Physiol 134:227-240.

Reinke H, Wild JM (1997) Distribution and connections of inspiratory premotor neurons in the brainstem of the pigeon (Columba livia). J Comp Neurol 379:347-362.

Reinke H, Wild JM (1998) Identification and connections of inspiratory premotor neurons in songbirds and budgerigar. J Comp Neurol 391:147-163.

Riede T, Suthers RA, Fletcher NH, Blevins WE (2006) Songbirds tune their vocal tract to the fundamental frequency of their song. Proc Natl Acad Sci U S A 103:5543-5548.

Striedter GF, Vu ET (1998) Bilateral feedback projections to the forebrain in the premotor network for singing in zebra finches. J Neurobiol 34:27-40.

Sturdy CB, Wild JM, Mooney R (2003) Respiratory and telencephalic modulation of vocal motor neurons in the zebra finch. J Neurosci 23:1072-1086.

Suthers RA, Zollinger SA (2004) Producing song: the vocal apparatus. Ann N Y Acad Sci 1016:109-129.

Suthers RA, Goller F, Wild JM (2002) Somatosensory feedback modulates the respiratory motor program of crystallized birdsong. Proc Natl Acad Sci U S A 99:5680-5685.

Tchernichovski O, Nottebohm F, Ho CE, Pesaran B, Mitra PP (2000) A procedure for an automated measurement of song similarity. Anim Behav 59:1167-1176.

Tremblay S, Shiller DM, Ostry DJ (2003) Somatosensory basis of speech production. Nature 423:866-869.

Whitney O, Soderstrom K, Johnson F (2000) Post-transcriptional regulation of zenk expression associated with zebra finch vocal development. Brain Res Mol Brain Res 80:279-290.

Wild JM (1981) Identification and localization of the motor nuclei and sensory projections of the glossopharyngeal, vagus, and hypoglossal nerves of the cockatoo (Cacatua roseicapilla), Cacatuidae. J Comp Neurol 203:351-377.

Wild JM (1990) Peripheral and central terminations of hypoglossal afferents innervating lingual tactile mechanoreceptor complexes in Fringillidae. J Comp Neurol 298:157-171.

Wild JM (1993a) Descending projections of the songbird nucleus robustus archistriatalis. J Comp Neurol 338:225-241.

Wild JM (1993b) The avian nucleus retroambigualis: a nucleus for breathing, singing and calling. Brain Res 606:319-324.

Wild JM (1994) Visual and somatosensory inputs to the avian song system via nucleus uvaeformis ( Uva) and a comparison with the projections of a similar thalamic nucleus in a nonsongbird, Columba livia. J Comp Neurol 349:512-535.

Wild JM (2004) Functional neuroanatomy of the sensorimotor control of singing. Ann N Y Acad Sci 1016:438-462.

Wild JM (2008) Birdsong: anatomical foundations and central mechanisms of sensorimotor integration. In: Neuroscience of Birdsong (Zeigler HP, Marler P, eds). Cambridge, UK: Cambridge UP.

Wild JM, Arends JJ (1987) A respiratory-vocal pathway in the brainstem of the pigeon. Brain Res 407:191-194.

Wild JM, Farabaugh SM (1996) Organization of afferent and efferent projections of the nucleus basalis prosencephali in a passerine, Taeniopygia guttata. J Comp Neurol 365:306-328. 
Wild JM, Krützfeldt NE (2012) Trigeminal and telencephalic projections to jaw and other upper vocal tract premotor neurons in songbirds: Sensorimotor circuitry for beak movements during singing. J Comp Neurol 520:590-605.

Wild JM, Zeigler HP (1980) Central representation and somatotopic organization of the jaw muscles within the facial and trigeminal nuclei of the pigeon (Columba livia). J Comp Neurol 192:175-201.

Wild JM, Zeigler HP (1996) Central projections and somatotopic organisation of trigeminal primary afferents in pigeon (Columba livia). J Comp Neurol 368:136-152.

Wild JM, Arends JJ, Zeigler HP (1984) A trigeminal sensorimotor circuit for pecking, grasping and feeding in the pigeon (Columba livia). Brain Res 300:146-151
Wild JM, Arends JJ, Zeigler HP (1985) Telencephalic connections of the trigeminal system in the pigeon (Columba livia): a trigeminal sensorimotor circuit. J Comp Neurol 234:441-464.

Wild JM, Arends JJ, Zeigler HP (1990) Projections of the parabrachial nucleus in the pigeon (Columba livia). J Comp Neurol 293:499523.

Wild JM, Li D, Eagleton C (1997) Projections of the dorsomedial nucleus of the intercollicular complex (DM) in relation to respiratory-vocal nuclei in the brainstem of pigeon (Columba livia) and zebra finch (Taeniopygia guttata). J Comp Neurol 377:392-413.

Williams H, Crane LA, Hale TK, Esposito MA, Nottebohm F (1992) Rightside dominance for song control in the zebra finch. J Neurobiol 23:10061020 . 